Received: 2020-05-14

Accepted: 2020-07-20 Published: 2021-01-31

\section{A Novel Approach to Brachial Plexus Injections: A Technical REPORT}

\author{
Myles Dworkin, MD, MPH' \\ lan Dworkin, MD² \\ Gerald Dworkin, DO
}

Background: Brachial plexus injects are common procedures for pain management of the upper limb. Complications from theses injections, however, can be severe leading to permanent neurological deficit. The purpose of this technical report is to introduces a safe novel approach for brachial plexus injections.

\title{
Case
}

Presentation: A 45-year-old woman with type 2 complex region pain disorder underwent a novel brachial plexus injection. The patient was brought to the operating room where fluoroscopy was used to locate the T2 lamina. A 17-gauge introducer needle was maneuvered through the interlaminar space and into the epidural space. The stylet was removed and a Brevi Kath® (Epimed, Dallas, TX) was passed through the C7-T1 foramen into the brachial plexus region. Contrast was used to ensure proper placement and absence of vascular pickup. The injection was then given, and the catheter withdrawn. The procedure was done without complication and the patient had a $40 \%$ reduction in pain symptoms based on a visual analog scale. She was cleared to restart physical therapy.

Conclusions: This novel technique approaches the brachial plexus by gaining access to the thoracic epidural space and following the spinal nerves through their respective foramen. The use of fluoroscopy and contrast ensures correct placement of injection. A blunt catheter limits the risk of injury. This approach may benefit patients who have failed standard techniques using local landmarks or those that are not ideal candidates due to previous injury or pre-existing conditions.

Key words: Interventional pain, injection, brachial plexus, pain management, fluoroscopy

\section{BACKGROUND}

Access to the brachial plexus is an important aspect of pain management of the upper limb. Safe application of injectable material to this area is essential for controlling upper extremity sensation, whether as a regional anesthesia technique during surgical procedures, or as a treatment modality for persistent pain symptoms (1-2). The surrounding anatomical structures, however, must be considered to ensure effective entrance into the perineural space. The brachial plexus originates as the $\mathrm{C} 5$ through $\mathrm{T} 1$ spinal nerve roots. These then form the ventral rami as they exit the spinal foramen and join together to form the superior (C5-C6), middle (C7), and inferior (C8-T1) trunks above the clavicle. The trunks pass under the clavicle in close proximity to each other before dividing distal to the

From: 'Sidney Kimmel Medical College of Thomas Jefferson University, Philadelphia, PA; ${ }^{2}$ Interventional Pain, Newport Care Medical Group, Newport Beach, CA; ${ }^{3}$ Department of Rehabilitation Medicine, Philadelphia College of Osteopathic Medicine, Philadelphia, PA

Corresponding Author: Myles Dworkin, MD, MPH $\quad$ E-mail: msd009@jefferson.edu

Disclaimer: There was no external funding in the preparation of this manuscript.

Conflict of interest: Each author certifies that he or she, or a member of his or her immediate family, has no commercial association (i.e., consultancies, stock ownership, equity interest, patent/licensing arrangements, etc.) that might pose a conflict of interest in connection with the submitted manuscript. 
clavicle and adjacent to the axillary artery into the lateral (C5-C7), posterior (C5-T1), and median (C8-T1) cords. Finally, the cords split into the terminal nerve branches including the musculocutaneous (C5-C6), median (C6-T1), ulnar (C8-T1), axillary (C5-C6), and radial (C5-T1) nerves in the axilla (2-3). The brachial plexus can be reached locally using several landmarks. Use of landmarks, however, has been associated with damage to surroundings (2).

Traditional approaches to the brachial plexus include the interscalene, supraclavicular, infraclavicular (anterior or retrograde), and axillary approaches. An isolated superior trunk block can also be performed through the supraclavicular fossa (2). Each approach accesses the brachial plexus at a different branching level, leading to anesthetic control of a different region between the shoulder and the fingertips. These techniques, however, become difficult in certain patient populations. Obesity may obscure local landmarks increasing the risk of injury to the brachial plexus or surrounding structures (4). Patients with pulmonary disease or poor pulmonary reserve are not good candidates due to the risk of pneumothorax (2). Furthermore, although the use of ultrasound and nerve stimulation may improve results and reduce complications, injuries to the nerve or surrounding structures may still occur associated with direct needle trauma (5-7). Posttraumatic injury may also interfere with normal neurofeedback needed for reliable stimulation monitoring as well as patient response to injections.

Complications associated with the common brachial plexus approaches are well known (8). These include neuropraxia, axonotmesis, and neurotmesis as well as injury to surrounding structures. The interscalene method places the ipsilateral phrenic nerve at risk as well as the vertebral artery. The supraclavicular approach risks damage to the subclavian artery and pneumothorax. Meanwhile, the infraclavicular approach jeopardizes the axillary artery and vein (2). Furthermore, patients with pain symptoms or recent trauma to the area may be reluctant to undergo local needle injections in this region, as this may lead to double crush syndrome (9-10).

The purpose of this article is to describe a novel minimally invasive approach to brachial plexus injections. This technique follows the cervical epidural and transforaminal space into the peripheral plexus region, and results in excellent neural proximity while minimizing complications associated with a direct, localized approach. A case highlighting the utility of this technique will be reviewed.

\section{CASE STUDY}

J.S. is a 45-year-old woman who suffered a fall at work resulting in significant trauma to her left shoulder. Xrays confirmed the presence of a fracture to her humeral head. The patient failed conservative treatment and was scheduled for a total shoulder replacement. Prior to the procedure, she was given a brachial plexus block for intraoperative and postsurgical pain control. Immediately upon providing the anesthesia, the patient felt a sharp, focal burning sensation that traveled distally through her left upper extremity. Although the pain soon mollified, the patient quickly developed intense and severe burning dysesthesias during the postoperative period consistent with complex regional pain disorder type 2, also known as causalgia.

At this time the patient was referred to an interventional pain specialist. Electromyographic evidence revealed lower brachial plexopathy. She underwent a number of treatments, including medical management and local injections, but failed to improve. The patient was unable to attend physical therapy or perform activities of daily living and therefore, a targeted brachial plexus injection procedure was indicated. We chose to perform a novel technique utilizing a Brevi-Kath (EpiMed, Dallas, TX) under fluoroscopic guidance.

\section{TECHNIQUE}

After obtaining consent, the patient was brought to the operating room and transferred in the prone position to a C-arm-compatible surgical table. Two pillows were positioned under the patient's chest providing subclavicular support. The neck was flexed with support provided to the forehead region. Intravenous anesthesia was provided including $100 \mathrm{mg}$ of midazolam and 100 $\mu \mathrm{g}$ of fentanyl. This provided pain control while allowing the patient to respond to questioning if needed. Sterile preparation was applied to the neck and upper posterior torso region.

C-arm fluoroscopy was utilized to locate the superior aspect of the T2 lamina on direct anteroposterior view contralateral to the patient's pain. We tilted caudally until the interlaminar space was open and tried to maximize the view of the T1-T2 interlaminar space. The point of entry was marked and $1 \%$ lidocaine, without epinephrine, was used for local anesthetic. We utilized a 25 -gauge, $3 \frac{1}{2}$-inch spinal needle to place lidocaine on the tip of the lamina. An Epimed 17-gauge introducer needle was then maneuvered under image-guidance to the interlaminar space using an entry angle between 
45 to 50 degrees with the skin's surface (Fig. 1). The needle was placed just off the inferior lamina on the side contralateral to the affected brachial plexus target.

The introducer needle was angled 20 to 30 degrees from the midline so as to point towards the ipsilateral C7-T1 foramen. Using a loss-of-resistance technique, we progressed the needle from the midpoint of the interlaminar space into the epidural space.

Following removal of the stylet, a Brevi Kath (Epimed, Dallas, TX) was then passed through the introducer needle and directed towards the C7-T1 foramen. This foramen provided excellent access to the lower trunk. A modest bend was placed at the end of the catheter which allowed us to navigate into and through the foramen into the brachial plexus region. The catheter was advanced to approximately $3 \mathrm{~cm}$ outside of the external foramen and into the inferior brachial plexus region (Fig. 2).

One $\mathrm{mL}$ of iopamidol 200 was used as a contrast medium to ensure correct placement of the needle as well as absence of vascular pickup (Fig. 3). At this point a solution with $1 \mathrm{~mL}$ of triamcinolone acetonide $40,1 \mathrm{~mL}$

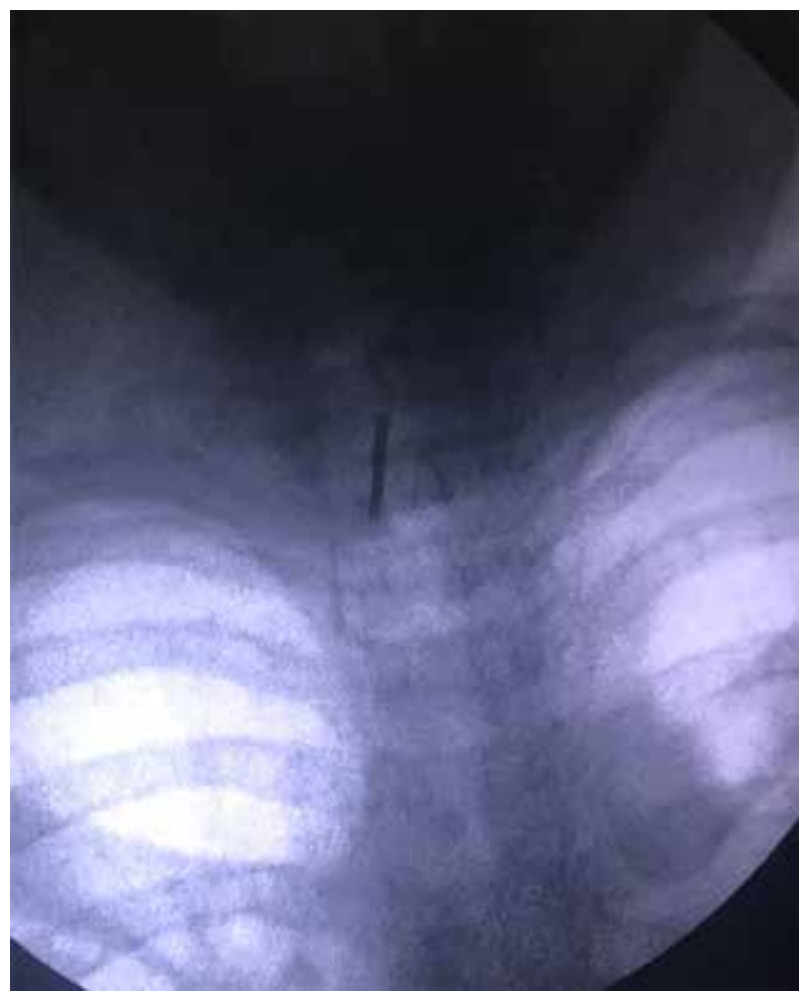

Fig. 1. Entry of introducer needle. of . $25 \%$ bupivacaine hydrochloride, and $3 \mathrm{~mL}$ of sterile saline was injected over several minutes. Following successful placement of the medication, the catheter was withdrawn as was the introducer needle. The patient was given a bandage and taken to the recovery room in stable condition.

\section{Patient Follow-Up}

The patient was seen 2 weeks following her procedure. She had a $40 \%$ reduction in pain symptoms based on a visual analog scale and was cleared to restart physical therapy. She was seen for repeat follow-up at 6 weeks post procedure. The patient continued to have reduced pain symptoms and was progressing in her therapy. She reported that she had begun performing basic activities of daily living and had increased independence.

\section{DISCUSSION}

This paper describes a novel approach to brachial plexus injections. Unlike standard approaches which access the peripheral nerves utilizing local landmarks in the neck and shoulder region, this technique approaches

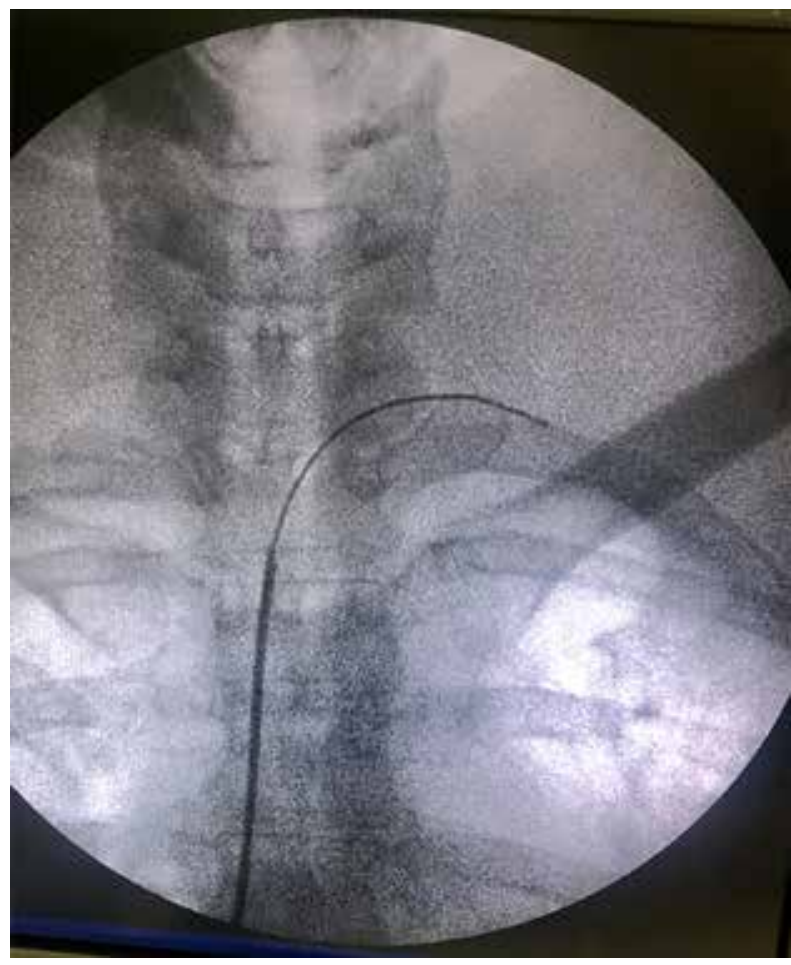

Fig. 2. Placement of catheter in inferior brachial plexus region. 


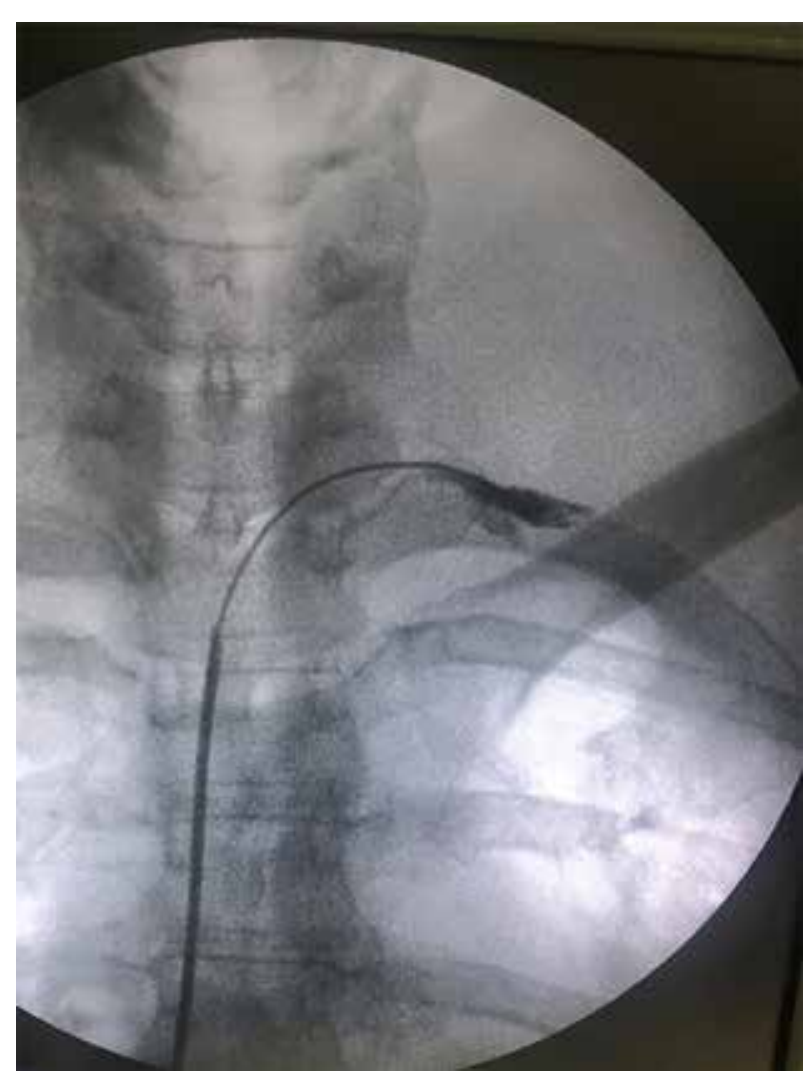

Fig. 3. Injection of contrast medium to ensure correct placement and absence of vascular pickup.

the brachial plexus by first gaining access to the epidural space and following the spinal nerves through their respective foramen. Fluoroscopic guidance ensures the correct placement of injectable material and the use of a blunt catheter limits nerve injury or damage to surrounding structures. The case reviewed in this paper demonstrates the use of this technique to access the inferior brachial plexus trunk. Superior and middle trunks may be accessed through the C5-C6 or C6-C7 foramen, respectively. To the authors' knowledge, this is the first paper to review this technique.

Complications related to peripheral nerve blocks are relatively rare with a reported incidence of about $3 \%$ within 4 to 6 weeks (7). When complications do occur, however, they can be severe leading to permanent sensory disturbance or motor loss with poor recovery (11). Efforts to reduce complications have yielded mixed results (12). While ultrasound guidance may reduce the rates of hemidiaphragmatic paresis, local anesthetic systemic toxicity, and pneumothorax, it has not been shown to significantly affect postoperative neurologic symptoms (13). Furthermore, the use of neurostimulation has not been shown to reduce complications compared to ultrasound alone (14). This has led to calls for the development of new techniques to provide peripheral nerve blocks (12). This paper describes one such technique that was used to achieve satisfactory pain control without complications in a patient who had previously failed alternative therapies.

The novel method reviewed in this paper may specifically benefit certain patients. Obese patients and those suffering from pulmonary disease are at increased risk of complications using standard techniques $(2,4)$. Using fluoroscopic guidance and approaching the brachial plexus from the epidural space, however, would minimize their risk. Additionally, patients with prior trauma to the area are often reluctant to have regional techniques and are predisposed to double-crush syndrome $(9,15)$. Posttraumatic nerve injury may also impair the effectiveness of neuromonitoring as well as impair patient feedback, increasing the risk of complication or failure. This novel technique provides an alternative approach for these patients and limits additional needle trauma to the area. Furthermore, peripheral nerve blocks are controversial in patients with certain neurological diseases such as multiple sclerosis (16). Using a blunt catheter and fluoroscopic guidance, as described in this paper, may be an attractive alternative for these patients.

This review has several limitations. While the purpose is to present and describe a novel technique, we review its use in only a single case. Furthermore, we only describe a brief postprocedural follow-up period. Although the patient was able to return to regular therapy and exhibited fewer pain symptoms, it is unknown whether the patient was able to return to full activities. The purpose of this paper, however, is not to prove the efficacy of brachial plexus injections but rather to describe a novel delivery technique which was performed without complications. The procedure has been utilized by our team in several additional cases with similar results. This case has been selected as it demonstrates a common patient encounter.

\section{CONCLUSION}

This technical note reviews a novel approach to brachial plexus injections. It utilizes a blunt catheter to enter through the epidural space and follows the spinal nerve through the spinal foramen. Using contrastenhanced fluoroscopy allows for localization of injectable material in the perineural space. This technique has 
been used to successfully manage a patient who had previously failed alternative treatments.

\section{Acknowledgment}

\section{Author Contributions}

MD - Author assisted with data acquisition, manuscript preparation, and approved final edits.

ID - Author assisted with project development, provided critical review, and approved final edits

GD - Author assisted with project development, provided critical review, and approved final edits.

Author access to data: All authors had access to chart documentation regarding the reported case and all data presented in the manuscript.

\section{Disclosures}

In consideration of the action of Interventional Pain Medicine Case Reports in reviewing and editing this submission (manuscript, tables, and figures), the author(s) undersigned hereby transfer(s), assign(s), or otherwise convey(s) all copyright ownership, including any and all rights incidental thereto, exclusively to ASIPP, in the event that such work is published.

\section{REFERENCES}

1. Mukherji SK, Wagle A, Armao DM, Dogra S. Brachial plexus nerve block with CT guidance for regional pain management: Initial results. Radiology 2000; 216:886-890.

2. Pester JM, Varacallo M. Brachial plexus block techniques. In: StatPearls [Internet]. Treasure Island, FL: StatPearls Publishing; 2019. www.ncbi.nlm.nih.gov/books/NBK470213/ Date Updated 04/26/2020. Date Accessed 05/02/2020.

3. Polcaro L, Daly DT. Anatomy, head and neck, brachial plexus. In: StatPearls [Internet]. Treasure Island, FL: StatPearls Publishing; 2020. https://pubmed.ncbi.nlm.nih.gov/30285368/. Date Updated 08/10/2020. Date Accessed 05/02/2020.

4. Nielsen KC, Guller U, Steele SM, Klein SM, Greengrass RA, Pietrobon R. Influence of obesity on surgical regional anesthesia in the ambulatory setting: An analysis of 9,038 blocks. Anesthesiology 2005; 102:181-187.

5. Raju PKB, Coventry DM. Ultrasound-guided brachial plexus blocks. BJA Educ 2014; 14:185-191.

6. Klaastad $\varnothing$, Sauter AR, Dodgson MS. Brachial plexus block with or without ultrasound guidance. Curr Opin Anaesthesiol 2009; 22:655-660.

7. Reiss W, Kurapati S, Shariat A, Hadzic A. Nerve injury complicating ultrasound/electrostimulation-guided supraclavicular brachial plexus block. Reg Anesth Pain Med 2010; 35:400-401.

8. Jeng $C L$, Torrillo TM, Rosenblatt MA. Complications of peripheral nerve blocks. Br J Anaesth 2010; 105:i97-i107.

9. Perretta DJ, Gotlin M, Brock K, et al. Brachial plexus blockade causes subclinical neuropathy: A prospective observational study. Hand (N Y) 2017; 12:50-54.

10. Hebl JR, Horlocker TT, Pritchard DJ. Diffuse brachial plexopathy after interscalene blockade in a patient receiving cisplatin chemotherapy: The pharmacologic double crush syndrome. Anesth Analg 2001; 92:249-251.

11. Brull R, McCartney CJ, Chan VW, El-Beheiry H. Neurological complications after regional anesthesia: Contemporary estimates of risk. Anesth Analg 2007; 104:965-974.

12. Kim HJ, Park SH, Shin HY, Choi YS. Brachial plexus injury as a complication after nerve block or vessel puncture. Korean J Pain $2014 ; 27: 210$

13. Neal JM. Ultrasound-guided regional anesthesia and patient safety: Update of an evidence-based analysis. Reg Anesth Pain Med 2016; 41:195-204.

14. Bayar I, Demir C, Sügür T, Karslı B, Inanoğlu K. The use of neurostimulation with ultrasound-guided brachial plexus block: Does it increase success? Agri 2019; 31:79-85.

15. Hebl JR, Horlocker TT, Pritchard DJ. Diffuse brachial plexopathy after interscalene blockade in a patient receiving cisplatin chemotherapy: The pharmacologic double crush syndrome. Anesth Analg 2001; 92:249-251.

16. Koff MD, Cohen JA, Mclntyre JJ, Carr CF, Sites BD. Severe brachial plexopathy after an ultrasound-guided single-injection nerve block for total shoulder arthroplasty in a patient with multiple sclerosis. Anesthesiology 2008; 108:325-328. 
